\title{
Alteration of Semantic Networks during Swear Words Processing in Schizophrenia
}

\author{
Sang Won Lee ${ }^{1, *}$, Bumseok Jeong ${ }^{1,2, *}$, Jong-II Park ${ }^{3,4}$, Gyung Ho Chung ${ }^{4,5}$, Hyo-Jong Lee ${ }^{6}$, Yin Cui ${ }^{3}$, \\ Woo-Sung $\mathrm{Kim}^{7}$, Kang Han $\mathrm{Oh}^{8}$, Il Seok $\mathrm{Oh}^{8}$, Guang Fan Shen ${ }^{3}$, Young-Chul Chung \\ ${ }^{1}$ Laboratory of Clinical Neuroscience and Development, Graduate School of Medical Science and Engineering, KAIST, ${ }^{2}$ Center of Optics for \\ Health Science, KAIST Institute, KAIST, Daejeon, Departments of ${ }^{3}$ Psychiatry and ${ }^{5}$ Radiology, Chonbuk National University Medical School, \\ ${ }^{4}$ Research Institute of Clinical Medicine of Chonbuk National University-Biomedical Research Institute of Chonbuk National University Hospital, \\ ${ }^{6}$ Department of Computer Science and Engineering \& Center for Advanced Image and Information Technology, 7 Department of Biomedical \\ Engineering, ${ }^{8}$ Division of Computer Science and Engineering, Chonbuk National University, Jeonju, Korea
}

\begin{abstract}
Objective: Positive symptoms, such as delusion and hallucination, commonly include negative emotional content in schizophrenia. We investigated the neural basis implicated during the processing of strong negative emotional words in patients with schizophrenia.

Methods: In our study, 35 patients with schizophrenia and 19 healthy controls were recruited, and the participants were asked to passively view the words that contained swearing and neutral content during functional magnetic resonance imaging.

Results: Patients with schizophrenia, compared to healthy controls, showed hypoactivation to the swear and neutral words stimuli in the left inferior frontal gyrus, left middle frontal gyrus, and left angular/supramarginal gyrus. More specifically, patients with remitted schizophrenia were found to have greater activation to the stimuli in the left middle/inferior frontal gyrus than patients with active schizophrenia. Furthermore, in the analysis of regions of interests, the left inferior and middle frontal gyrus activity was related to the severity of positive symptoms, including delusion and suspiciousness. Conclusion: Our results suggest that patients with schizophrenia have difficulty in semantic processing and inhibitory control of swear words, and these abnormalities may be connected with the severity of positive symptoms.
\end{abstract}

KEY WORDS: Schizophrenia; Semantic network; Swear words; Inferior frontal gyrus; Positive symptom; Middle frontal gyrus.

\section{INTRODUCTION}

Impaired emotional functioning has been considered as one of the fundamental symptoms of schizophrenia. Most of the studies using various emotional paradigms reported emotion perception deficits. ${ }^{1)}$ The deficits are associated with impairments in processing of emotion perception/recognition, ${ }^{1,2)}$ emotional memory, ${ }^{3)}$ emotional salience attribution, ${ }^{4)}$ and emotion regulation. ${ }^{5,6)}$ Emotion

Received: July 10, 2017 / Revised: September 22, 2017

Accepted: October 14, 2017

Address for correspondence: Young-Chul Chung, MD, PhD

Department of Psychiatry, Chonbuk National University Medical

School, 20 Geonji-ro, Deokjin-gu, Jeonju 54907, Korea

Tel: +82-63-250-2185, Fax: +82-63-275-3157

E-mail: chungyc@jbnu.ac.kr

ORCID: https://orcid.org/0000-0001-9491-1822

*These authors contributed equally to this work. perception abilities are related to social competence ${ }^{7,8)}$ and predict later work functioning and independent living. ${ }^{9)}$ However, it should be noted that studies using real-world experience sampling methodology ${ }^{10,11)}$ observed higher negative emotionality in patients with schizophrenia compared to controls.

Functional magnetic resonance imaging (fMRI) studies have identified several brain regions associated with impaired emotional functioning. A recent meta-analysis supports findings of overall reduced amygdala activation in response to facial emotion processing in schizophrenia. ${ }^{12)}$ There are, however, opposite findings, i.e., increased activation in the amygdala in response to threatening faces ${ }^{13)}$ and emotional words ${ }^{14)}$ in schizophrenia. Another important region is the frontal lobe. Less activation in the ventrolateral prefrontal cortex (VLPFC) during reappraisal of negative affect ${ }^{6)}$ and decreased activation of the pre-

(c) This is an Open-Access article distributed under the terms of the Creative Commons Attribution Non-Commercial License (http://creativecommons.org/licenses/by-nc/4.0) which permits unrestricted non-commercial use, distribution, and reproduction in any medium, provided the original work is properly cited. 
frontal-parietal network during response inhibition to negative words ${ }^{15)}$ were observed in patients with schizophrenia compared to healthy controls. These findings indicate impairment of cognitive control of negative emotion in schizophrenia. In addition, anomalies in the semantic network in response to negative emotional words were reported in schizophrenia. ${ }^{16)}$ This finding is interesting in that the role of semantic network is to facilitate semantic integration between concepts, and impairment in semantic association is observed in schizophrenia. ${ }^{17)}$

Swear words contain the persecutory contents that can induce fear, hostility, and be related to insult. ${ }^{18)}$ Auditory verbal hallucinations often contain swearing and threatening words. ${ }^{19)}$ Patients with psychotic disorders also had a higher negative emotional valence of the content of auditory hallucination than healthy individuals with auditory hallucination symptoms. ${ }^{20)}$ Persecutory delusions often include negative, threatening, and derogatory content. ${ }^{21)}$ In this regard, exploring the neural correlates involved in processing swear words would be a nice tool to investigate pathogenesis of delusion or auditory hallucinations. To the best of our knowledge, there have been no studies evaluating the functional brain changes while processing swear words in patients with schizophrenia.

We hypothesized that neural response to negative emotional words would be different in patients with schizophrenia compared to controls and this impairment could be different between active and remitted schizophrenia patients. The aims of the study were to explore alterations in brain network during exposure to swear words and its relationship with positive symptoms in patients with active and remitted schizophrenia.

\section{METHODS}

\section{Subjects}

In total, 35 patients with schizophrenia (mean age,
$35.1 \pm 10.4$ years; 17 females) and 19 controls (mean age, $34.5 \pm 8.4$ years; 11 females) participated in the study. Among 35 patients with schizophrenia, 21 were a remitted state, and 13 had active symptoms of schizophrenia. One patient who finished the fMRI test did not participate in our clinical evaluation. Based on the previous study, ${ }^{22)}$ we defined the remitted state as no rating $\geq 4$ (moderate) on any of 4 psychotic items (delusion, suspiciousness/persecution, hallucinatory behavior, conceptual disorganization) of the Positive and Negative Syndrome Scale (PANSS) for 2 months. All patients met the criteria of the Diagnostic and Statistical Manual of Mental Disorders 4th edition, text revision (DSM-IV-TR) for schizophrenia, according to the Structured Clinical Interview for DSM-IV (SCID). ${ }^{23)}$ Severity of symptoms was evaluated with PANSS $^{24)}$ and the Clinical Global Impression-Schizophrenia scale $(\mathrm{CGI}-\mathrm{SCH})^{25)}$ by two psychiatrists. All control subjects were screened for Axis I psychiatric disorders using the SCID. Detailed characteristics of the subjects and statistical comparisons between the patients with schizophrenia and the controls are described in Table 1. All subjects joined this study voluntarily and provided a written informed consent. The study was approved by the Ethics Committee of Chonbuk National University Hospital (No. CUH 2012-08-001).

\section{Preliminary Words Selection for fMRI Experiment}

Seventy swear words were selected by the national survey on language use and language attitude in adolescents in Korea ${ }^{26)}$ and 70 neutral words were chosen from two references based on valence, arousal, and dominance scores. ${ }^{27,28)}$ Neutral words were translated into Korean. We standardized all words stimuli by asking 15 healthy participants to rate a list of 70 words on ease of understanding and frequency on an 11-point visual analogue scale (0 to 10). First, words that achieved a score of 7 or higher with ease of understanding were selected. Then,

Table 1. Characteristics of the participants (all schizophrenia and control)

\begin{tabular}{lccc}
\multicolumn{1}{c}{ Variable } & Schizophrenia (all) $(\mathrm{n}=35)$ & Control $(\mathrm{n}=19)$ & $p$ value \\
\hline Age $(\mathrm{yr})$ & $35.8 \pm 10.5$ & $33.9 \pm 8.2$ & $0.51^{\dagger}$ \\
Education (yr) & $12.6 \pm 2.2$ & $16.9 \pm 3.3$ & $<0.001^{\dagger}$ \\
Sex, male/female & $20 / 15$ & $8 / 11$ & $0.29^{\dagger}$ \\
Handedness*, right/left & $32 / 1$ & $17 / 0$ & $0.47^{\dagger}$ \\
\hline
\end{tabular}

Values are presented as mean \pm standard deviation or number only.

*Not performed for two participants.

${ }^{\dagger}$ Independent $t$ test, ${ }^{\ddagger}$ chi-square test. 
40 swear words (Supplementary Table 1; available at online only) and 40 neutral words were selected based on the frequency. For swear words, scores of the frequency were 6.05 and 6.15 for the session 1 and 2 of the scanning. For neutral words, scores of the frequency were 5.41 and 5.18 for the session 1 and 2 of the scanning. There was no significant differences in frequency between swear words and neutral words in both session 1 $(p=0.259)$ and session $2(p=0.082)$.

\section{fMRI Experiment}

One day before fMRI scan, all participants practiced the fMRI task off-line with a small number of words (10 stimuli). In the fMRI session, the task followed an event-related design. Twenty swear words and 20 neutral words were used, and each stimulus was presented for three seconds in pseudorandom order. Also, 26 fixation crosses that jittered between 3 and 9 seconds were applied between the stimuli. Some words in the conditions were presented continuously without a fixation cross (for example, swearing word $\rightarrow$ neutral word). The duration of a session was 4 minutes and 36 seconds, and two sessions with T1-weighted scans in between were conducted. Participants were asked to passively view the words and press 'yes' for swear words, and 'no' for neutral words using a button-press. The behavioral response was intended to maintain the participant's attention.

After the fMRI experiment, the subjects rated the emotional valences of the swear words and neutral words using an 11-point Likert scale (0-10 for swear words [10 represents extremely negative] and from -5 to 5 for neutral words [ -5 is extremely negative]). Using different scales, two-side for neutral words and one-side for swear words, can be confounding factor. However, it is difficult to measure the emotional valence of neutral words using one-side scale because the meanings of neutral words may vary from person to person. Therefore, we matched a range of Likert scale (11 point), and absolute value was applied to negative values of neutral words. The absolute values of the Likert scale of neutral words were multiplied by two. Then, the ratio of the mean value of the emotional valence between the swear words and neutral words was calculated using the following equation; Mean value of emotional valence of swear words/Mean absolute emotional value of neutral words $\times 2$. In the estimation of the hemodynamic response function (HRF), swear word stim- uli were weighted with a ratio of 2.7 .

\section{Image Acquisition and Preprocessing}

A total of 184 (92×2 sessions) blood oxygen level dependent (BOLD) based fMRI images were acquired at 3.0 Tesla (Semens Verio, Erlangen, Germany) using a $\mathrm{T} 2 *$-weighted gradient echo, echo-planar imaging (EPI) pulse sequence (36 axial slices; repetition time $[T R]=3,000 \mathrm{~ms}$, echo time $(T E)=30 \mathrm{~ms}$, flip angle $=90^{\circ}$, field of view $[\mathrm{FOV}]=240 \times 240 \mathrm{~mm}$, acquisition matrix $=64 \times 64$, slice thickness $=3 \mathrm{~mm}$ with a gap of $0.75 \mathrm{~mm}$, voxel size $=3.75 \times 3.75 \times 3.0 \mathrm{~mm})$. A high resolution structural image was obtained with a T1 weighted sequence (176 sagittal slices; TR=1,900 ms, TE=2.5 ms, flip angle $=9^{\circ}, \mathrm{FOV}=250 \times 250 \mathrm{~mm}$, slice thickness $=1 \mathrm{~mm}$, voxel size $=0.5 \times 1 \times 0.5 \mathrm{~mm}$ ) between two fMRI sessions.

The BOLD images were pre-processed with the fMRI Expert Analysis Tool (FEAT) of the FMRIB Software Library (FSL). ${ }^{29)}$ Motion correction was performed using the linear interpolation method (MCFLIRT). Skull stripping of the functional images was conducted by a brain extraction tool (BET). Spatial smoothing using a $5 \mathrm{~mm}$ full-width/ half-maximum Gaussian Kernel and a high pass filter (cutoff frequency 100 seconds) were applied. Spatial registration of functional EPI images to T1 weighted anatomical volumes was conducted with boundary-based registration, $^{30)}$ and T1 weighted anatomical images were coregistered to Montreal Neurological Institute (MNI) standard space using FMRIB's Nonlinear Image Registration Tool (FNIRT).

\section{fMRI Analysis}

The FSL's FEAT was used for the first-, second-, and third-level analyses. In first-level analysis, because of insufficient interstimulus intervals, we made a single regressor including swear and neutral words stimuli since some of words stimuli were presented continuously without an interstimulus interval. To apply an appropriate emotional weight on swear words, three column format (starting second, duration of stimuli, and weight of the stimuli for first, second and third column, respectively) in FSL was used. Swear word stimuli were 2.7-times weighted compared to neutral word stimuli based on the valence scores of a post fMRI survey. In addition, analyses using equal, 2, and 4 times weight on swear word stimuli were conducted to support our findings. The stimuli were 
convolved with a gamma HRF. A general linear model (GLM) was applied to measure the activities of brain regions in a single subject level analysis. In second-level group analyses, parameter estimates of two sessions were combined using a fixed-effects model. In third-level group analyses, an unpaired $t$ test using a mixed-effects model in the FSL (FLAME 1) was performed for comparing the parameter estimates of the schizophrenia subjects and the control subjects. In addition, a three-group analysis using an analysis of variance (ANOVA) was used to measure the differences among active, remitted schizophrenia and control. In this study, cluster-based correction for multiple comparison was applied using Gaussian random field theory, with a cluster-forming threshold of $Z>2.3$. The threshold for cluster significance was a family-wise error (FWE) corrected $p<0.05$, and this $p$ value threshold was used in all the other analyses in our study. Age, gender, and amount of education were included as covariates in the group comparison analyses.

\section{Correlation between Regions of Interest (ROI) and Symptom Severity}

ROls that showed group differences were used to study the correlation between the brain activity and symptoms. Although alteration in the right hemisphere semantic network was also possible, many studies showed abnormalities in the left hemisphere semantic network rather than right semantic network. ${ }^{31)}$ Also, our group comparison analysis (control $>$ schizophrenia) showed alterations in left semantic network. Therefore, we chose five left hemisphere ROls that play an important role in the semantic system and have had significant results in the analyses of group differences between schizophrenia and controls. The ROIs were made using the sphere (radius $5 \mathrm{~mm}$ ) around the peak MNI coordinate of parameter estimate maps in control > schizophrenia (Table 2). ${ }^{32)}$ The five ROIs (and $\mathrm{MNI}$ peak coordinates) were left frontal pole $(-42 / 46 / 10)$, left middle frontal gyrus $(-52 / 14 / 40)$, left angular gyrus $(-44 /-54 / 50)$, left supramarginal gyrus $(-56 /-46 / 50)$, and left inferior frontal gyrus $(-54 / 30 / 2)$. Partial correlation coefficients using non-parametric method (Spearman) were estimated between symptoms of schizophrenia and parameters in the activation maps of schizophrenia patients. Age, gender, and amount of education were included as covariates in the analyses. SPSS Statistics release ver. 20 (IBM, Somers, NY, USA) was used for the statistical analysis.

\section{RESULTS}

\section{Sample Characteristics and Group Comparisons of Valance Ratings of Task Stimuli}

Educational years, but not gender distribution, age and handedness, showed significant differences between the

Table 2. Characteristics of the participants (active, remitted schizophrenia [SCZ], and control)

\begin{tabular}{|c|c|c|c|c|c|c|c|}
\hline \multirow{2}{*}{ Variable } & \multicolumn{2}{|c|}{ SCZ (active) } & \multicolumn{2}{|c|}{ SCZ (remitted) } & \multicolumn{2}{|c|}{ Control } & \multirow{2}{*}{$p$ value } \\
\hline & Data & $\mathrm{N}$ & Data & $\mathrm{N}$ & Data & $\mathrm{N}$ & \\
\hline Age (yr) & $39.8 \pm 11.2$ & 13 & $32.7 \pm 9.2$ & 21 & $33.9 \pm 8.2$ & 19 & $0.09^{\dagger, \neq}$ \\
\hline Education (yr) & $12.1 \pm 2.3$ & 13 & $12.9 \pm 2.1$ & 21 & $16.9 \pm 3.3^{q}$ & 19 & $<0.001^{\ddagger}$ \\
\hline Sex, male/female & $10 / 3$ & 13 & $9 / 12$ & 21 & $8 / 11$ & 19 & $0.10^{\S}$ \\
\hline Handedness, right/left & $12 / 0$ & $12^{*}$ & $20 / 1$ & 21 & $17 / 0$ & $17^{*}$ & $0.49^{\S}$ \\
\hline PANSS-Total & $56.5 \pm 9.5$ & 13 & $40.1 \pm 7.9$ & 21 & & & $<0.001^{\|}$ \\
\hline PANSS-Positive & $14.2 \pm 3.8$ & 13 & $8.6 \pm 1.8$ & 21 & & & $<0.001^{\|}$ \\
\hline PANSS-Negative & $15.2 \pm 4.7$ & 13 & $10.7 \pm 3.7$ & 21 & & & $0.004^{\|}$ \\
\hline PANSS-General & $27.2 \pm 7.2$ & 13 & $20.9 \pm 4.0$ & 21 & & & $0.002^{\|}$ \\
\hline CGI-SCH-Total & $4.2 \pm 1.0$ & 13 & $2.7 \pm 0.9$ & 21 & & & $<0.001^{\|}$ \\
\hline CGI-SCH-Positive & $4.0 \pm 0.8$ & 13 & $2.1 \pm 1.0$ & 21 & & & $<0.001^{\|}$ \\
\hline CGI-SCH-Negative & $3.0 \pm 1.0$ & 13 & $2.1 \pm 0.9$ & 21 & & & $0.012^{\|}$ \\
\hline Chlorpromazine equivalents $^{\dagger}$ & $419.4 \pm 371.3$ & $12^{*}$ & $513.5 \pm 324.2$ & $20^{*}$ & & & $0.457^{\|}$ \\
\hline Duration of illness (mo) & $158.0 \pm 109.0$ & $12^{*}$ & $83.5 \pm 60.7$ & 21 & & & $0.016^{\|}$ \\
\hline
\end{tabular}

Values are presented as mean \pm standard deviation or number only.

PANSS, Positive and Negative Syndrome Scale; CGI-SCH, Clinical Global Impression-Schizophrenia scale.

*Not performed for two participants.

${ }^{\dagger}$ Calculated based on Hales $(2008)^{32)}$; ${ }^{\ddagger}$ one way analysis of variance test, ${ }^{\S}$ chi-square test, and "independent $t$ test; " significantly higher value than others in post-hoc analysis using Bonferroni correction (all $p<0.001$ ). 

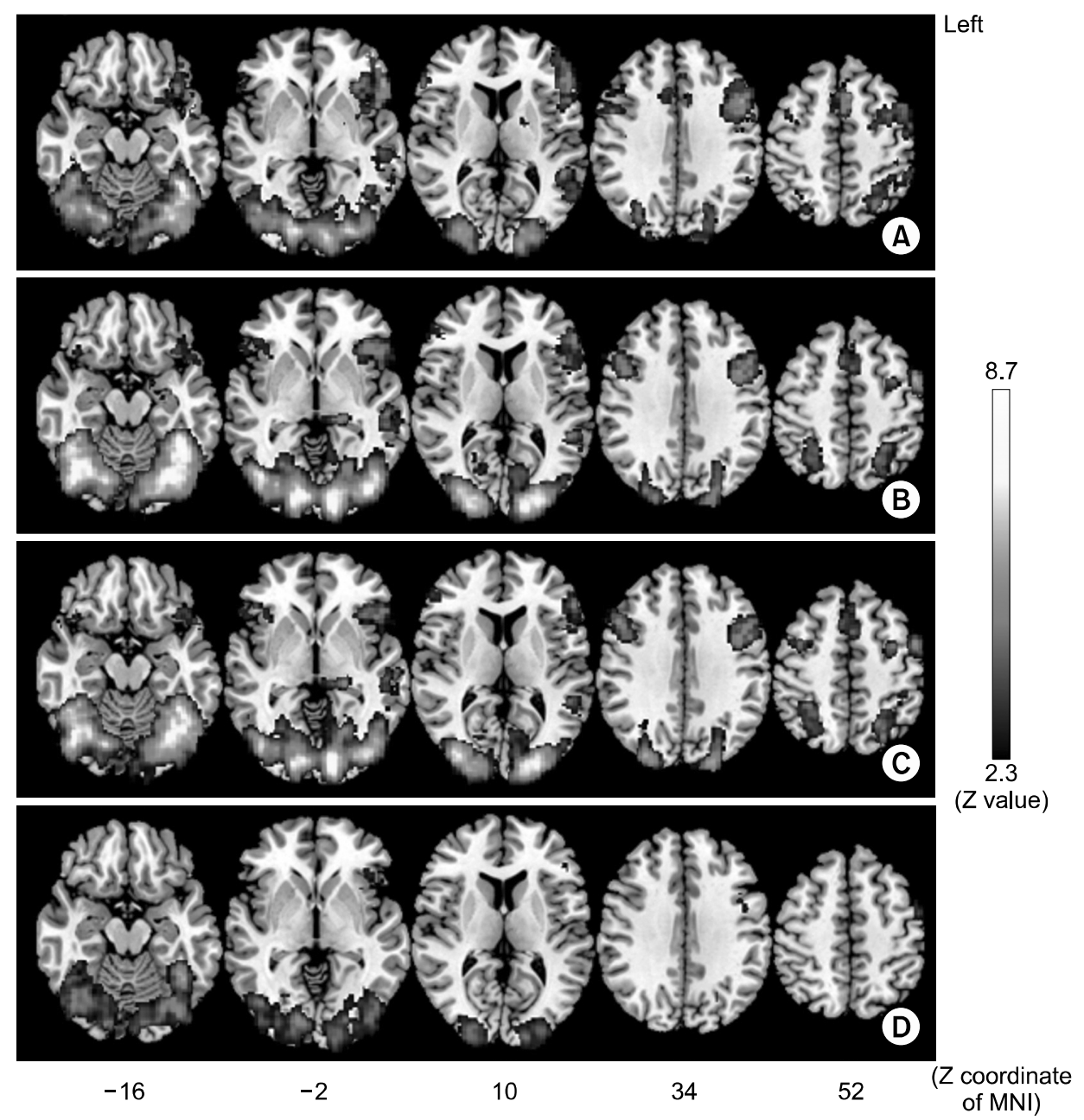

Fig. 1. Group mean activity maps in (A) control, (B) all schizophrenia (remitted+active), (C) remitted, and (D) active groups $(Z>2.3$, familywise error corrected $p<0.05)$.

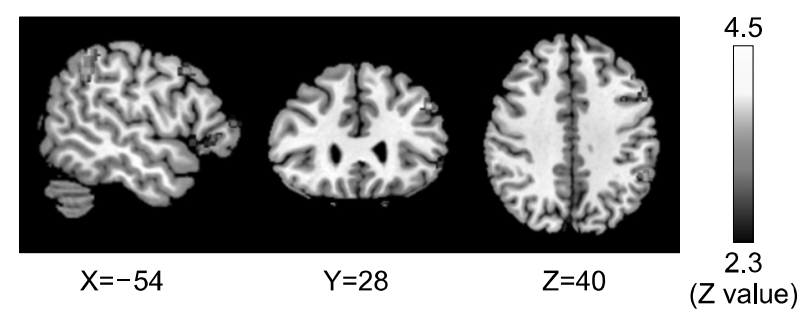

Fig. 2. Group differences between all schizophrenia and control (Z $>2.3$, family-wise error corrected $p<0.05$; one-way ANOVA and post-hoc analyses). Control $>$ all schizophrenia (remitted+active).

control group and the all schizophrenia group (Table 1). Detailed results of sample characteristics are described in Table 2. Furthermore, 34 patients with schizophrenia and 17 controls finished the post-fMRI survey. Among the whole group, the mean valence score of swear words (5.45) was higher than the absolute mean valence score of neutral words (1.01). The mean valence scores of swear words were 5.43 (standard deviation [SD], 3.4) for schizophrenia and 5.49 (SD, 2.7) for controls, and those of neutral words were $0.70(\mathrm{SD}, 1.3)$ for schizophrenia and 0.25 (SD, 0.4) for controls. There was no significant difference in emotional valence of swear words and neutral words in the survey between the control group and the schizophrenia group ( $\mathrm{t}=-0.056, p=0.955$ for swear words and $\mathrm{t}=1.333, p=0.189$ for neutral words).

\section{Group Differences between Patients and Controls}

During fMRI scanning, both the schizophrenia group and the control group showed more significant activities in various brain regions, including the bilateral middle frontal gyrus, the left inferior frontal gyrus, the bilateral lateral occipital cortex (LOC), and the left angular gyrus (Fig. $1 \mathrm{~A}, 1 \mathrm{~B})$. Within these regions, the left inferior frontal gyrus, the left middle frontal gyrus and the left angular gyrus were more activated in the control than in the schizo- 
Table 3. The differences in brain activities between patients with schizophrenia and healthy controls

\begin{tabular}{|c|c|c|c|c|c|}
\hline \multirow{2}{*}{$\begin{array}{c}\text { Brain region } \\
\text { (control }>\text { schizophrenia) }\end{array}$} & \multirow{2}{*}{ Cluster size } & \multicolumn{3}{|c|}{ Peak voxel coordinates in MNI space } & \multirow{2}{*}{$Z$ value } \\
\hline & & $\mathrm{x}$ & y & $\mathrm{z}$ & \\
\hline Left frontal pole & 209 & -42 & 46 & 10 & 3.70 \\
\hline Left middle frontal gyrus & 188 & -52 & 14 & 40 & 3.64 \\
\hline Left angular gyrus & 183 & -44 & -54 & 50 & 3.92 \\
\hline Left supramarginal gyrus & & -56 & -46 & 50 & 3.73 \\
\hline Left inferior frontal gyrus & 95 & -54 & 30 & 2 & 3.02 \\
\hline Left middle frontal gyrus & 60 & -48 & 30 & 36 & 3.89 \\
\hline
\end{tabular}

Cluster size is the number of voxels and clusters consisted of 20 or more were included. Threshold is family-wise error -corrected $p<0.05$.
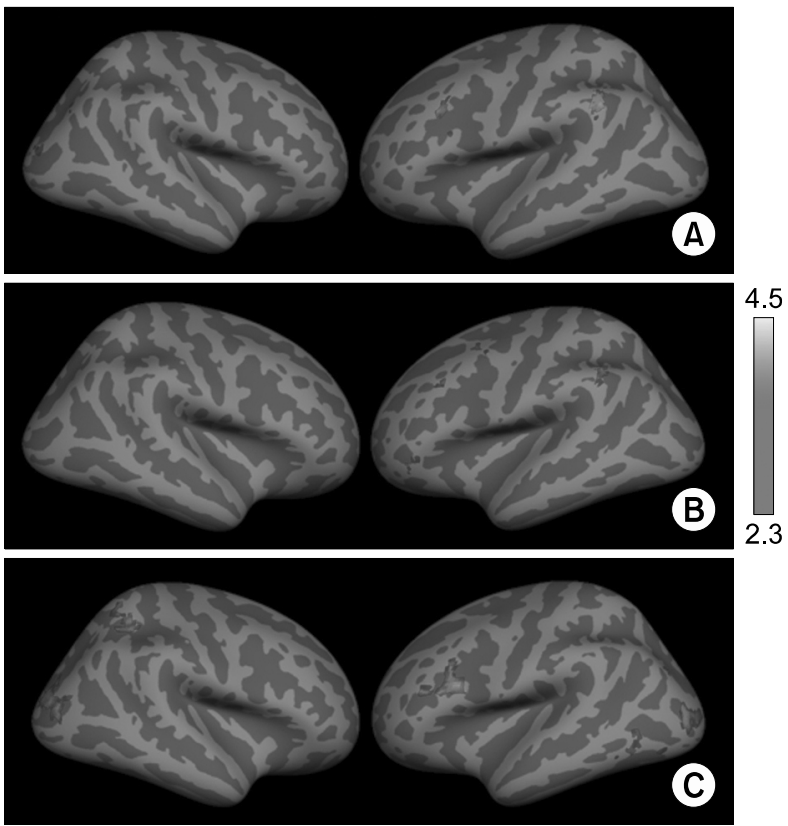

Fig. 3. Brain regions that showed differences in activity during the task between groups $(Z>2.3$, family-wise error corrected $p<0.05$; one-way ANOVA and post-hoc analyses). (A) Control $>$ active schizophrenia; (B) control>remitted schizophrenia; (C) remitted schizophrenia $>$ active schizophrenia.

phrenia group (corrected $p<0.05$ ) (Fig. 2, Table 3). The results of subtraction contrast (activation condition [swear words]-neutral condition) revealed no significant difference between patients and controls.

\section{Differences in Brain Activities among Patients with Active or Remitted Schizophrenia and Healthy Controls}

The overall activity patterns of patients with remitted schizophrenia were similar to that of healthy controls, but were different from that of patients with active schizophrenia (Fig. 1). Both patients with remitted schizophrenia and healthy controls had activation in the bilateral in- ferior frontal gyrus, the bilateral middle frontal gyrus, the left dorsomedial prefrontal gyrus (DMPFC), the bilateral LOC, and the left angular gyrus. In contrast, active schizophrenia subjects only showed activities in the bilateral LOC, the left inferior frontal gyrus and the left precentral gyrus (Fig. 1). One-way ANOVA and post-hoc analyses showed that both subjects with remitted and active schizophrenia had significantly lower activities in the left middle frontal gyrus and the left supramarginal gyrus compared to the control (corrected $p<0.05$ ). In addition, activities of the DMPFC and bilateral LOC were lower in the active schizophrenia group than in the control group. Among the patients with schizophrenia, the remitted group had a higher level of activities in the bilateral middle frontal gyrus, bilateral inferior frontal gyrus, bilateral LOC, and right superior parietal lobule (corrected $p<0.05$ ). These differences remained the same after the duration of illness was further included as covariate (data not shown). Figures 2 and 3 illustrates the results of the differences. In addition, a cluster including the left middle/inferior frontal gyrus were over activated in remitted schizophrenia compared to active schizophrenia regardless of the weights on swear words (Fig. 4).

\section{Correlation with Symptoms of Schizophrenia}

In whole brain correlation analyses, there was no brain region that showed a significant correlation with the scores of PANSS-Positive (including subscales), Negative, General, and Total (corrected $p>0.05$ ).

In a partial (Spearman) correlation analysis after controlling for the effects of age, gender, and amount of education, the activity in the ROI of the left inferior frontal gyrus showed significant correlations with the scores of PANSS-Total ( $r=-0.400, p=0.026)$ and with subscales scores of PANSS-Positive: delusion ( $r=-0.502, p=0.004)$, 
Swearing:neutral $=1: 1$
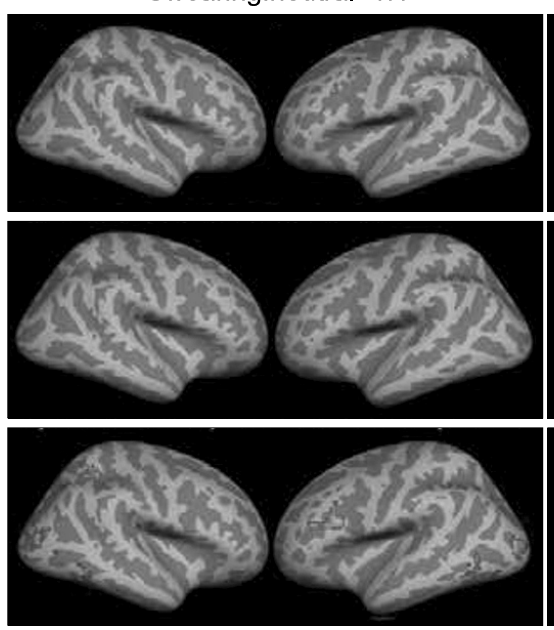

Swearing:neutral $=2: 1$
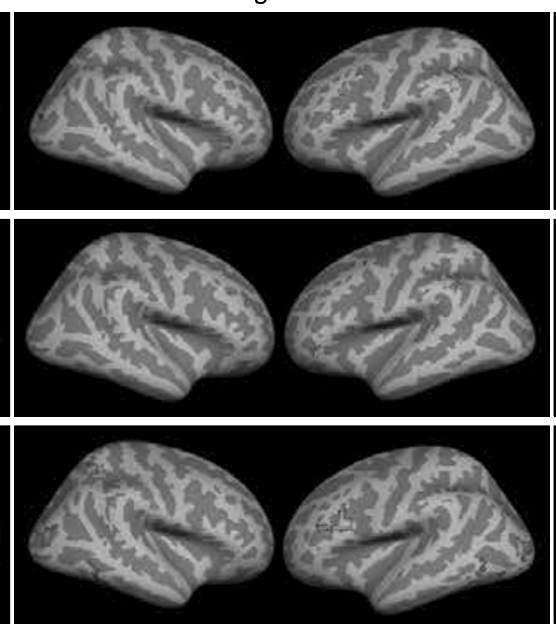

Swearing: neutral $=4: 1$

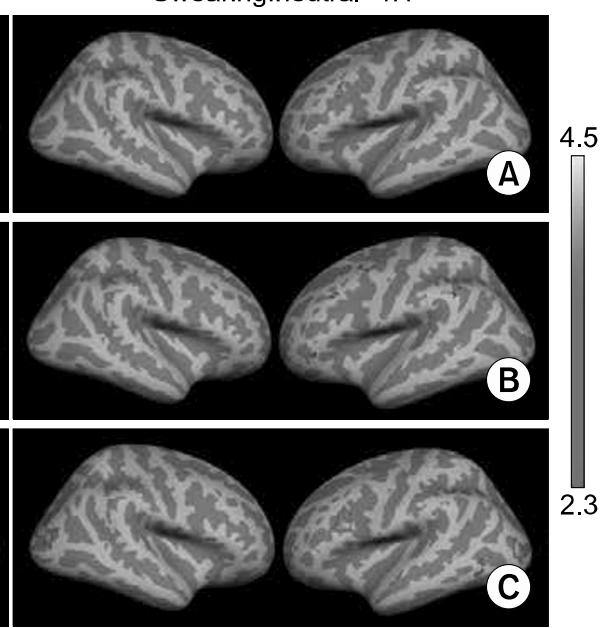

Fig. 4. Group differences in brain activities according to different weights on swearing stimuli $(Z>2.3$, family-wise error corrected $p<0.05)$. (A) Control > active schizophrenia, (B) control > remitted schizophrenia, (C) remitted schizophrenia > active schizophrenia.
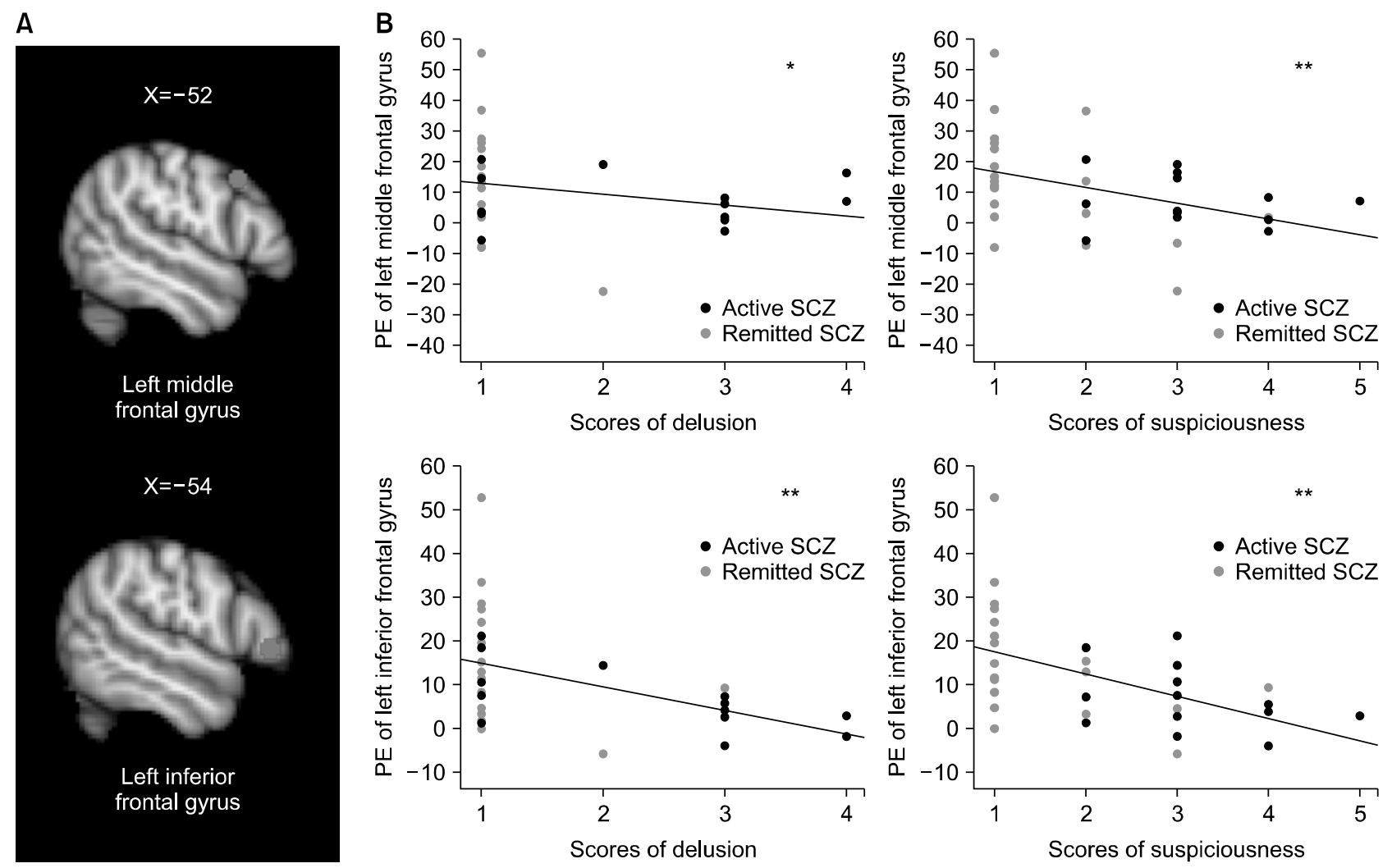

Fig. 5. The regions of interest of left middle/inferior frontal gyrus and left inferior gyrus (A), and the relationships between 'Positive' scores of the Positive and Negative Syndrome Scale and parameter estimates of regions of interest (B). SCZ, schizophrenia; PE, parameter estimates. ${ }^{*} p<0.05$ and ${ }^{* *} p<0.01$.

and suspiciousness/persecution ( $\mathrm{r}=-0.518, p=0.003)$. Furthermore, the activity of the left middle frontal gyrus was negatively correlated with the scores of PANSS-
Positive subscale scores: delusion $(r=-0.373, p=0.039)$ and suspiciousness/persecution ( $r=-0.512, p=0.003)$ (Fig. 5). The other ROls did not show any significant rela- 
tionships with the scores of PANSS-Total, PANSS-Positive, PANSS-Negative, and PANSS-General (all $p$ values $>0.05$ ).

\section{DISCUSSION}

We observed no significant differences in the participants' rating of emotional valence between swear words and neutral words in the survey, and in amygdala activation between the control group and the schizophrenia group. These were unexpected findings given that mostly underactivation $^{12)}$ or in few cases overactivation ${ }^{13,14)}$ of amygdala in response to emotional processing has been reported. Instead, we found underactivation of the angular/supramarginal gyrus and left inferior and middle frontal cortex, parts of the semantic network, ${ }^{33)}$ in patients with schizophrenia compared to controls.

While patients with schizophrenia reported subjective emotional valence ratings of swear words similar to healthy controls, differences in the activities of several brain regions were found. The angular/supramarginal gyrus, which is included in the association cortex was consistently more activated in the control in both two-group and threegroup analysis in our study. Considering same underactivation of the angular/supramarginal gyrus in both remitted and active schizophrenia, an abnormality of the left angular gyrus can be a sustainable trait marker of schizophrenia. The angular/supramarginal gyrus is involved in various mental processes, including semantic processing, word comprehension, and social cognition. ${ }^{34)}$ In a word reading activity like our experimental paradigm, the activity of the left angular/supramarginal gyrus was commonly found in healthy subjects ${ }^{34,35)}$; this activity was closely connected to semantic processing. ${ }^{36)}$ The function of the left angular gyrus may take a top hierarchy in the integration of semantic information and retrieval of underlying concepts. Several studies on schizophrenia presented both structural and functional abnormalities in this region. A reduced volume in the left angular gyrus in first onset schizophrenia ${ }^{37)}$ and rightward asymmetry of the angular gyrus in chronic schizophrenia were reported. ${ }^{38)}$ In fMRI studies, patients with chronic schizophrenia had low activity of the supramarginal gyrus during semantic repetition priming ${ }^{39)}$ and a reduced functional connectivity of the left inferior frontal gyrus-left supramarginal gyrus during semantic encoding. ${ }^{40)}$ Therefore, our findings suggest that patients with schizophrenia may have difficulties in understanding the meaning of negative stimuli (swear words) and integrating those with previous experiences rather than in processing emotional reaction to swear words.

Furthermore, the schizophrenia group showed lower activity in left inferior and middle frontal cortex than control. In addition, although the sample size of active schizophrenia was rather small, within the schizophrenia groups, remitted schizophrenia subjects presented a higher activity in the cluster of left prefrontal cortex and left occipital cortex than patients with active schizophrenia. The function of the left inferior frontal gyrus was related to efficiency of semantic processing, integration of linguistic meaning with world knowledge, ${ }^{41)}$ and inhibitory control. ${ }^{15,42)}$ Under-activation of the left inferior frontal gyrus ${ }^{40,43)}$ and discordance of the activities between the left inferior frontal cortex and the left inferior parietal cortex were found in patients with schizophrenia during semantic encoding. ${ }^{43)}$ Moreover, the deficits in the mirror neuron system ${ }^{44,45)}$ are frequently reported in schizophrenia. Both inferior frontal gyrus and inferior parietal lobule are important brain regions in the mirror neuron system. The mentalizing impairment resulting from failure in observation-execution matching in the mirror neuron system leads to symptoms of reality distortion including inferring false intentions, delusional ideation, and misinterpretation of reality ${ }^{46)}$ which refer to auditory hallucination, delusion, ${ }^{44)}$ and failed emotional processing. ${ }^{47)}$ Taken together, our results of low activation in inferior and middle frontal cortex in schizophrenia indicate that patients with schizophrenia may have difficulties in semantic processing of swear words and inhibitory control to swear words. Assuming that inhibitory response to negative stimuli is a defense mechanism, patients with schizophrenia would be more vulnerable to negative stimuli (swear words) because of weakening or deficit in controlling negative stimuli.

As for the correlation results, only the left prefrontal cortex involving the left inferior frontal gyrus, not the supramarginal/angular gyrus showed a relationship to the severity of positive symptoms. This prefrontal structure, which interacts with the temporal and visual cortex, ${ }^{48)}$ plays an important role in inhibiting responses to negative words $^{15,42)}$ and controlling the semantic process via a top-down mechanism. ${ }^{33)}$ A previous study reported a negative correlation between the activities of the left inferior frontal cortex during semantic processing and the severity of formal thought disorder. ${ }^{49)}$ Our results suggest that al- 
terations in control networks, rather than integration networks, during semantic processing with strong negative emotional values may be related to positive symptoms.

There are several limitations in our study. First, as some of the stimuli were presented continuously without an interstimulus interval, it was difficult to make the HRF for each condition. Alternative way was to apply an appropriate emotional weight on swear words. We only reported the results that were consistent with the analysis using various emotional weights (Fig. 4). Second, the amount of education was significantly different between groups. Even though we obtained similar results after controlling for education, matched samples would have produced different results. Third, we cannot match lengths of words between swearing and neutral words because our words set was relatively small. Finally, our results between patients and controls might have been caused by medication. To disentangle medication effects, recruiting drugnaïve or drug-free patients should be considered in future studies. In spite of these limitations, to the best of our knowledge, while many studies have been done to clarify the abnormalities of emotional and semantic processing networks in schizophrenia, this is the first study to reveal the differences in brain activity during swear words with strong negative emotional values that can be related to symptoms of schizophrenia.

In conclusion, our results demonstrated that patients with schizophrenia show altered activation in angular/ supramarginal gyrus and inferior and middle frontal cortex, in response to swear words. The left inferior frontal gyrus, not the supramarginal/angular gyrus was found to be associated with the severity of positive symptoms. Further studies should be followed to explore the exact meaning of semantic processing impairment of swear words and its mechanisms leading to the formation of positive symptoms.

\section{Acknowledgments}

This study was supported by a grant from the Korean Health Technology R\&D Project, Ministry of Health \& Welfare, Republic of Korea (HI13C1459) and also supported by grant for KAIST Future Systems Healthcare Project from the Ministry of Education, Science and Technology (N10150017, N01150030 to B Jeong).

\section{REFERENCES}

1. Kohler CG, Walker JB, Martin EA, Healey KM, Moberg PJ. Facial emotion perception in schizophrenia: a meta-analytic review. Schizophr Bull 2010;36:1009-1019.

2. Habel U, Chechko N, Pauly K, Koch K, Backes V, Seiferth N, et al. Neural correlates of emotion recognition in schizophrenia. Schizophr Res 2010;122:113-123.

3. Herbener ES. Emotional memory in schizophrenia. Schizophr Bull 2008;34:875-887.

4. Lee SK, Chun JW, Lee JS, Park HJ, Jung YC, Seok JH, et al. Abnormal neural processing during emotional salience attribution of affective asymmetry in patients with schizophrenia. PLoS One 2014;9:e90792.

5. Strauss GP, Kappenman ES, Culbreth AJ, Catalano LT, Lee BG, Gold JM. Emotion regulation abnormalities in schizophrenia: cognitive change strategies fail to decrease the neural response to unpleasant stimuli. Schizophr Bull 2013;39: 872-883.

6. van der Meer L, Swart M, van der Velde J, Pijnenborg G, Wiersma D, Bruggeman R, et al. Neural correlates of emotion regulation in patients with schizophrenia and non-affected siblings. PLoS One 2014;9:e99667.

7. Hooker C, Park S. Emotion processing and its relationship to social functioning in schizophrenia patients. Psychiatry Res 2002;112:41-50.

8. Mueser KT, Doonan R, Penn DL, Blanchard JJ, Bellack AS, Nishith $\mathrm{P}$, et al. Emotion recognition and social competence in chronic schizophrenia. J Abnorm Psychol 1996; 105:271-275.

9. Kee KS, Green MF, Mintz J, Brekke JS. Is emotion processing a predictor of functional outcome in schizophrenia? Schizophr Bull 2003;29:487-497.

10. Myin-Germeys I, Delespaul PA, deVries MW. Schizophrenia patients are more emotionally active than is assumed based on their behavior. Schizophr Bull 2000;26:847-854.

11. Myin-Germeys I, Krabbendam L, Delespaul PA, van Os J. Sex differences in emotional reactivity to daily life stress in psychosis. J Clin Psychiatry 2004;65:805-809.

12. Li H, Chan RC, McAlonan GM, Gong QY. Facial emotion processing in schizophrenia: a meta-analysis of functional neuroimaging data. Schizophr Bull 2010;36:1029-1039.

13. Satterthwaite TD, Wolf DH, Loughead J, Ruparel K, Valdez JN, Siegel SJ, et al. Association of enhanced limbic response to threat with decreased cortical facial recognition memory response in schizophrenia. Am J Psychiatry 2010;167:418-426.

14. Sanjuan J, Lull JJ, Aguilar EJ, Martí-Bonmatí L, Moratal D, Gonzalez JC, et al. Emotional words induce enhanced brain activity in schizophrenic patients with auditory hallucinations. Psychiatry Res 2007;154:21-29.

15. Vercammen A, Morris R, Green MJ, Lenroot R, Kulkarni J, Carr $\mathrm{VJ}$, et al. Reduced neural activity of the prefrontal cognitive control circuitry during response inhibition to negative words in people with schizophrenia. J Psychiatry Neurosci 2012;37: 379-388. 
16. Klumpp H, Keller J, Miller GA, Casas BR, Best JL, Deldin PJ. Semantic processing of emotional words in depression and schizophrenia. Int J Psychophysiol 2010;75:211-215.

17. Jamadar SD, Pearlson GD, O'Neil KM, Assaf M. Semantic association fMRI impairments represent a potential schizophrenia biomarker. Schizophr Res 2013;145:20-26.

18. Vingerhoets AJJM, Bylsma LM, de Vlam C. Swearing: A biopsychosocial perspective. Psychological Topics 2013;22:287-304.

19. Upthegrove R, Ives J, Broome MR, Caldwell K, Wood SJ, Oyebode F. Auditory verbal hallucinations in first-episode psychosis: a phenomenological investigation. BJPsych Open 2016;2:88-95.

20. Daalman K, Boks MP, Diederen KM, de Weijer AD, Blom JD, Kahn RS, et al. The same or different? A phenomenological comparison of auditory verbal hallucinations in healthy and psychotic individuals. J Clin Psychiatry 2011;72:320-325.

21. Freeman D. Suspicious minds: the psychology of persecutory delusions. Clin Psychol Rev 2007;27:425-457.

22. Larsen TK, Moe LC, Vibe-Hansen L, Johannessen JO. Premorbid functioning versus duration of untreated psychosis in 1 year outcome in first-episode psychosis. Schizophr Res 2000;45:1-9.

23. First MB. Structured clinical interview for DSM-IV-TR Axis I disorders: Patient edition. New York:Biometrics Research Department, Columbia University;2005.

24. Kay SR, Fiszbein A, Opler LA. The positive and negative syndrome scale (PANSS) for schizophrenia. Schizophr Bull 1987;13:261-276.

25. Haro JM, Kamath SA, Ochoa S, Novick D, Rele K, Fargas A, et al. The Clinical Global Impression-Schizophrenia scale: a simple instrument to measure the diversity of symptoms present in schizophrenia. Acta Psychiatr Scand Suppl 2003;(416): 16-23.

26. Chang K. A national survey on language use and language attitude in adolescents. Seoul:The National Institute of The Korean Language;2011.

27. Toglia MP, Battig WF. Handbook of semantic word norms. Hillsdale, NJ: Erlbaum;1978.

28. Bradley MM, Lang PJ. Affective Norms for English Words (ANEW): Instruction manual and affective ratings. Gainesville: University of Florida, Center for Research in Psychophysiology; 1999.

29. Jenkinson $M$, Beckmann CF, Behrens TE, Woolrich MW, Smith SM. FSL. Neuroimage 2012;62:782-790.

30. Greve DN, Fischl B. Accurate and robust brain image alignment using boundary-based registration. Neuroimage 2009; 48:63-72.

31. Rapp AM, Steinhäuser AE. Functional MRI of sentence-level language comprehension in schizophrenia: a coordinatebased analysis. Schizophr Res 2013;150:107-113.

32. Hales RE, Yudofsky SC, Roberts LW. The American psychiatric publishing textbook of psychiatry. 5th ed. Washington, D.C.:American Psychiatric Pub;2008.
33. Lau EF, Phillips C, Poeppel D. A cortical network for semantics: (de)constructing the N400. Nat Rev Neurosci 2008;9: 920-933.

34. Seghier ML. The angular gyrus: multiple functions and multiple subdivisions. Neuroscientist 2013;19:43-61.

35. Houdé O, Rossi S, Lubin A, Joliot M. Mapping numerical processing, reading, and executive functions in the developing brain: an fMRI meta-analysis of 52 studies including 842 children. Dev Sci 2010;13:876-885.

36. Price CJ, Mechelli A. Reading and reading disturbance. Curr Opin Neurobiol 2005; 15:231-238.

37. Nierenberg J, Salisbury DF, Levitt JJ, David EA, McCarley RW, Shenton ME. Reduced left angular gyrus volume in first-episode schizophrenia. Am J Psychiatry 2005;162:1539-1541.

38. Niznikiewicz M, Donnino R, McCarley RW, Nestor PG, Iosifescu DV, O'Donnell B, et al. Abnormal angular gyrus asymmetry in schizophrenia. Am J Psychiatry 2000;157: 428-437.

39. Jeong B, Kubicki M. Reduced task-related suppression during semantic repetition priming in schizophrenia. Psychiatry Res 2010;181:114-120.

40. Jeong B, Wible CG, Hashimoto R, Kubicki M. Functional and anatomical connectivity abnormalities in left inferior frontal gyrus in schizophrenia. Hum Brain Mapp 2009;30:41384151.

41. Hagoort P, Hald L, Bastiaansen M, Petersson KM. Integration of word meaning and world knowledge in language comprehension. Science 2004;304:438-441.

42. Swick D, Ashley V, Turken AU. Left inferior frontal gyrus is critical for response inhibition. BMC Neurosci 2008;9:102.

43. Kubicki M, McCarley RW, Nestor PG, Huh T, Kikinis R, Shenton ME, et al. An fMRI study of semantic processing in men with schizophrenia. Neuroimage 2003;20:1923-1933.

44. Arbib MA, Mundhenk TN. Schizophrenia and the mirror system: an essay. Neuropsychologia 2005:43:268-280.

45. Tseng CE, Chien YL, Liu CM, Wang HL, Hwu HG, Tseng WY. Altered cortical structures and tract integrity of the mirror neuron system in association with symptoms of schizophrenia. Psychiatry Res 2015;231:286-291.

46. Sprong M, Schothorst P, Vos E, Hox J, van Engeland H. Theory of mind in schizophrenia: meta-analysis. Br J Psychiatry 2007;191:5-13.

47. Aleman A, Kahn RS. Strange feelings: do amygdala abnormalities dysregulate the emotional brain in schizophrenia? Prog Neurobiol 2005;77:283-298.

48. Moritz-Gasser S, Herbet G, Duffau H. Integrating emotional valence and semantics in the human ventral stream: a hodological account. Front Psychol 2015;6:32.

49. Borofsky LA, McNealy K, Siddarth P, Wu KN, Dapretto M, Caplan R. Semantic processing and thought disorder in childhood-onset schizophrenia: Insights from fMRI. J Neurolinguistics 2010;23:204-222. 